\title{
Daumen hoch für neue Rhizarthrose-Prothese
}

Eine speziell geformte Endoprothese mit variabler Kopfgröße soll die Probleme überwinden helfen, vor denen die endoprothetische Versorgung arthrotischer Daumensattelgelenke in den vergangenen Jahrzehnten gestanden hat.

eit bald 50 Jahren wird versucht, das Prinzip, arthrotische Gelenke mit einer Endoprothese zu versorgen, auch auf das Sattelgelenk zwischen Os metacarpale I und Os trapezium zu übertragen. Allerhand Materialien wechselten sich dabei ab: Silikon, Keramik, Polyethylen, Pyrocarbon und Metall. Doch die Probleme wie Versagen, Lockerung oder Subluxation des Implantats blieben erhalten.

US-Orthopäden haben nun 138 Patienten mit Rhizarthrose eine neue Prothesenvariante eingesetzt, die Revisionseingriffe vermeiden helfen soll: das BioPro Modular Thumb Implant. Der Schaft der Kobalt-Chrom-Prothese steht in adduzierender Varusstellung, um Dislokationen entgegenzuwirken. Ein Baukastensystem sorgt dafür, dass der halbkugelige Gelenkkopf passend aufgesetzt werden kann. Sowohl die Höhe wie auch die Breite der Hemisphäre können an den Patienten angepasst werden.

Die im Zuge der Studie behandelten Patienten litten an Rhizarthrose in den Stadien II oder III nach Eaton-Littler; Stadium III bedeutet dabei eine signifikante Gelenkdestruktion mit Zysten, Sklerose, Subluxation und mehr als $2 \mathrm{~mm}$ messenden Osteophyten. Das Durchschnittsalter der Betroffenen lag bei 63 Jahren, 78\% von ihnen waren Frauen. Geschädigte Anteile der Gelenkfläche und die Basis des Metacarpale I wurden reseziert, der Schaft der Prothese in den Markraum eingeschlagen und anschließend ein in der Größe zur Gelenkfläche passender Kopf auf den Schaft aufgesetzt.

Auswertbar waren die Daten von 124 Patienten mit 143 operierten Daumensattelgelenken. Bei der abschließenden Follow-up-Untersuchung, im Mittel 72 (Bereich: 35-120) Monate nach dem Eingriff, hatte sich die Schmerzsituation bei 135 und die Funktionalität von 138 Gelenken gebessert. 139 Daumen wurden von ihren Besitzern in der Gesamteinschätzung mit „sehr gut“ oder "gut“ bewertet. Die Kraftentwicklung im Spitzgriff hatte sich von durchschnittlich 4,9 kg auf 6,4 kg gesteigert. Der postoperative Buck-GramckoScore, der im optimalen Fall 56 Punkte beträgt, stand bei 49 Punkten. Insgesamt mussten nur sechs Revisionseingriffe vorgenommen werden, 94\% der Implantate waren nach dem Follow-up noch intakt.

Fazit: Mit der speziell geformten, mit passgenauem Kopf ausrüstbaren Endoprothese BioPro ${ }^{\varpi}$ Modular Thumb Implant für den Ersatz des Daumensattelgelenks wurden in der vorliegenden Studie zur Rhizarthrose überzeugende Resultate erzielt. Probleme wie Dislokation oder Implantatversagen traten nur in einem geringen Prozentsatz auf. Ob sich das Verfahren auch für andere Indikationen eignet, beispielsweise posttraumatische oder entzündliche Arthritiden des Trapeziometakarpalgelenks, muss aber offen bleiben.

Dr. Robert Bublak

Pritchett JW, Habryl LS. A Promising Thumb Basal Joint Hemiarthroplasty for Treatment of Trapeziometacarpal Osteoarthritis. Clin Orthop Relat Res 2012; 470: 2756-63

Kommentar: Die operative Therapie der Daumensattelgelenkarthrose stellt Chirurgen auch heute noch teilweise vor besondere Herausforderungen. Die Vielzahl verschiedener Konzepte - von der Resektionsarthroplastik über die Versteifung bis hin zum Gelenkersatz - belegen diese Si- tuation. Angesichts der hohen Zahl von Rhizarthrose-Patienten in der täglichen Praxis ist die Entwicklung weiterer Operationsmöglichkeiten daher wichtig, um jedem Betroffenen eine individuelle Therapie anbieten zu können.

Der vorliegende Arbeit von Prichett beschreibt die Erfahrungen mit einer neu entwickelten Hemiprothese. Für eine erste Auswertung waren die Fallzahlen hoch genug. Nach den bisherigen Ergebnissen scheint das vorgestellte Verfahren durchaus geeignet, sich in das vorhandene Therapieportfolio gleichwertig einzureihen.

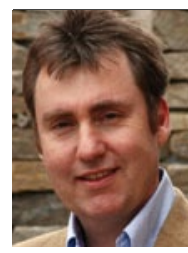

Dr. med. Michael Strassmair Zentrum für Handchirurgie am Klinikum Starnberg Osswaldstrasse 1 82319 Starnberg www.handchirurgie-starnberg.de 